CURVATURA MÉDIA DE FOLHEAÇÕES TRANSVERSALMENTE ORIENTÁVEIS

André de Oliveira Gomes

TESE APRESENTADA

AO

INSTITUTO DE MATEMÁTICA E ESTATÍSTICA

DA

UNIVERSIDADE DE SÃO PAULO

PARA OBTENÇÃO DO GRAU DE DOUTOR

EM

MATEMÁTICA

Área de Concentração: Geometria Diferencial

Orientador: Prof. Dr. Fabiano Gustavo Braga Brito

-São Paulo, Junho de 2003- 


\section{CURVATURA MÉDIA DE FOLHEACÕES TRANSVERSALMENTE ORIENTÁVEIS}

Este exemplar corresponde à redação final da tese devidamente corrigida e defendida por André de Oliveira Gomes e aprovada pela comissão julgadora

São Paulo, 11 de junho de 2003.

Banca Examinadora:

- Prof.Dr. Fabiano Gustavo Braga Brito (IME-USP)

- Prof.Dr. José Antonio Verderesi (IME-USP)

- Prof.Dr. Francesco Mercuri (IMECC-UNICAMP)

- Prof.Dr. Luiz Amancio Machado Souza Junior (UNIRIO)

- Prof.Dr. Sebastião Carneiro de Almeida (UFCe) 


\title{
Resumo
}

Neste trabalho, estudamos uma folheação $F$ de classe $C^{\infty}$ e codimensão um definida sobre uma variedade riemanniana $M$. Assumimos que $F$ é transversalmente orientável. Sob esta hipótese, mostramos que a função curvatura média de $F$ tem um limite superior. Usando este resultado, determinamos uma condição necessária e suficiente para que a folheação seja totalmente geodésica. Estudamos folheações rotacionais no espaço hiperbólico e mostramos que tais folheações não existem na 3 -esfera euclidiana.

\begin{abstract}
In this work we study a codimension-one $C^{\infty}$-foliation $F$ of a complete riemannian manifold $M$. We assume that $F$ is transversely orientable. Under this hypothesis, we show that the mean curvature function of $F$ has a superior limit. Using this result, we find a necessary and sufficient condition for the foliation $F$ to be totally geodesic. We study rotational foliations of the hyperbolic space and we show that there are no rotational foliations of the euclidean 3-sphere.
\end{abstract}




\section{Introdução.}

O estudo dos aspectos geométricos de uma folheação de codimensão um definida sobre uma variedade Riemanniana, segue uma tradição iniciada por Reinhart [12] e posteriormente desenvolvida por Rummler [14], Sullivan [15], Harvey-Lawson [6] e mais recentemente por Oshikiri [9], [10] e [11]. A idéia é utilizar os conceitos e técnicas da geometria Riemanniana e aplicá-los ao estudo da geometria das folheações. Deste modo, noções como a de segunda forma fundamental, função curvatura média, curvatura de Ricci etc., são fácilmente estendidas para folheações de uma variedade Riemanniana. Por outro lado, é conhecido desde os trabalhos de Abe [1], o importante papel desempenhado pelas equações diferenciais do tipo Riccati no estudo de tais folheações.

Assim, no Capítulo 1, após uma breve exposição dos conceitos básicos, apresentamos uma aplicação da equação de Riccati ao estudo das folheações transversalmente orientáveis de uma variedade Riemanni- 
ana completa, mostrando que a função curvatura média de uma tal folheação tem um limite superior dado em função da curvatura de Ricci e do divergente. Como corolário, determinamos uma condição necessária e suficiente para que uma tal folheação seja totalmente geodésica. Exibimos também alguns exemplos de folheações no espaço hiperbólico e determinamos uma condição suficiente para que uma tal folheação, definida em uma variedade de curvatura seccional constante, possua folhas totalmente umbílicas.

No Capítulo 2, utilizamos técnicas da geometria equivariante para determinar quais são as folheações do espaço hiperbólico que são rotacionais, i.e., folheações que são invariantes pela ação de um grupo ortogonal e cujas folhas possuem curvatura média constante. Mostramos ainda que tais folheações não existem na 3-esfera euclidiana.

Finalmente, no Capítulo 3 aplicamos os resultados desenvolvidos nos capítulos anteriores ao estudo das folheações Riemannianas e obtemos como corolário algumas proposições demonstradas em TondeurVanhecke [19] para o caso de codimensão um. 


\section{Conteúdo}

1 Folheações Transversalmente Orientáveis 3

1.1 Introdução. . . . . . . . . . . . . . . . . . . 3

1.2 Preliminares. . . . . . . . . . . . . . . 4

1.3 Resultados Principais. . . . . . . . . . . . . . 8

1.4 Exemplos no Espaço Hiperbólico. . . . . . . . . . . 15

1.5 Folheações Totalmente Umbílicas. . . . . . . . . . . . 18

2 Folheações Rotacionais $\quad 20$

2.1 Introdução. . . . . . . . . . . . . . . 20

2.2 Preliminares. . . . . . . . . . . . . . . . . . . . 21

2.3 Um sistema de coordenadas para $\mathbb{H}^{3} / G$. . . . . . . 23

2.4 Folheações do espaço hiperbólico $\mathbb{H}^{3}$. . . . . . . . 25 
2.5 A não-existência de uma folheação rotacional na esfera euclidiana $\mathbb{S}^{3} \ldots \ldots \ldots \ldots$

3 Folheações Riemannianas 33

3.1 Introdução. . . . . . . . . . . . . . . . . . . . 33

3.2 Preliminares. . . . . . . . . . . . . . . . 33

3.3 Resultados Principais . . . . . . . . . . . . . . . 36

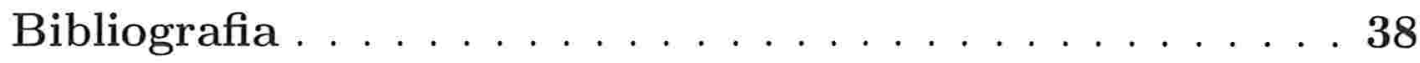




\section{Capítulo 1}

\section{Folheações Transversalmente Orientáveis}

\subsection{Introdução.}

Neste Capítulo, mostraremos como definir a segunda forma fundamental e a curvatura média de uma folheação definida sobre uma variedade riemanniana e provaremos que se a folheação for transversalmente orientável, isto é, se existe um campo unitário global que é ortogonal às folhas da folheação, a curvatura média possui um limite superior. Como corolário, obtemos uma condição necessária e suficiente para que a folheação seja totalmente geodésica, ou seja, para que cada folha da folheação seja uma subvariedade totalmente geodésica da variedade ambiente. Exibimos alguns exemplos de tais folheações no 
espaço hiperbólico e determinamos uma condição suficiente para que uma folheação transversalmente orientável, definida em um espaço de curvatura constante, tenha folhas totalmente umbílicas.

\subsection{Preliminares.}

Em todo este capítulo, $M^{n+1}$ vai representar uma variedade riemanniana simplesmente conexa, orientável, de classe $C^{\infty}$, completa e de dimensão $n+1$ munida de uma métrica $\langle$,$\rangle . Os símbolos \nabla$ e $R$ vão representar a conexão e a curvatura de $M$ respectivamente. Vamos ainda considerar definida sobre $M$ folheações de classe $C^{\infty}$ e de codimensão um que denotaremos por $F$. Vamos supor que todas as funções e campos utilizados são de classe $C^{\infty}$.

1.2.1 Definição. Uma folheação $F$ de codimensão um definida sobre uma variedade riemanniana completa $M$ é dita Transversalmente Orientável se existe um campo de vetores unitário $N$, definido sobre toda a $M$, que é ortogonal a cada folha da folheação $F$.

Em particular, cada folha da folheação $F$ é uma subvariedade orientável de $M$. Seja então $F$ uma folheação transversalmente orientável de $M$ e considere um referencial ortonormal local adaptado dado por 


$$
\left\{e_{1}, e_{2}, \ldots, e_{n}, e_{n+1}\right\}
$$

definido em uma vizinhança de $M$. Então, os vetores $e_{1}, e_{2}, \ldots, e_{n}$ são tangentes às folhas de $F$ enquanto que o vetor $e_{n+1}=N$ é ortogonal às folhas de $F$. No que segue, usaremos a seguinte convenção para a variação dos índices

$$
1 \leq A, B, C, \ldots \leq n+1 \quad \text { e } \quad 1 \leq i, j, k, \ldots \leq n
$$

Seja $\left\{w_{1}, w_{2}, \ldots, w_{n}, w_{n+1}\right\}$ o referencial dual correspondente, isto é,

$$
w_{A}\left(e_{B}\right)=\delta_{A B}
$$

Temos ainda as 1-formas de conexão, denotadas por $w_{A B}$, definidas pela equação

$$
\nabla_{X} e_{A}=\sum w_{A B}(X) e_{B}
$$

Pelas equações de estrutura, obtemos

$$
\begin{aligned}
& d w_{A}=\sum w_{B} \wedge w_{B A} \\
& d w_{A B}=\sum w_{A C} \wedge w_{C B}+\Omega_{A B}
\end{aligned}
$$

onde, por definição, $\Omega_{A B}=-\frac{1}{2} \sum R_{A B C D} w_{C} \wedge w_{D}$. Definido sobre a variedade $M$ temos o Tensor de Ricci dado por 


$$
\operatorname{Ric}(N)=\frac{1}{n} \sum R_{n+1 i n+1 i}=\frac{1}{n} \sum\left\langle R\left(N, e_{i}\right) N, e_{i}\right\rangle
$$

Sejam agora, $\theta_{A}$ e $\theta_{A B}$ as restrições das formas $w_{A}$ e $w_{A B}$ em vetores tangentes às folhas da folheação $F$. Então é claro que

$$
\theta_{n+1}=0, \quad \theta_{i}=w_{i} \quad \text { e } \quad \theta_{n+1 i}=-\sum h_{i j} \theta_{j}
$$

Assim, temos $\sum \theta_{n+1 i} \wedge \theta_{i}=0$ e portanto, pelo Lema de Cartan, obtemos que $h_{i j}=h_{j i}$. Por outro lado, a Segunda Forma fundamental das folhas é dada por

$$
B=\sum h_{i j} \theta_{i} \otimes \theta_{j}
$$

e a sua norma é dada por $\|B\|^{2}=\sum h_{i j}^{2}$. A Função Curvatura Média é

$$
H=\frac{1}{n} \sum h_{i i}
$$

e o Divergente de um campo de vetores $V$ definido sobre $M$ é dado localmente por

$$
\operatorname{div}(V)=\sum\left\langle\nabla_{e_{A}} V, e_{A}\right\rangle
$$


1.2.2 Proposição.[3] Seja F uma folheação de classe $C^{\infty}$ de codimensão um e transversalmente orientável definida sobre a variedade riemanniana completa $M^{n+1}$ e seja $N$ um campo de vetores unitário ortogonal às folhas de $F$ definido em toda a variedade $M$. Então, temos

$$
\operatorname{div}\left(\nabla_{N} N\right)=-n d H(N)+\|B\|^{2}+n \operatorname{Ric}(N)
$$

Demonstração. Utilizando o referencial ortonormal adaptado $\left\{e_{A}\right\}$, temos que

$$
w_{n+1 i}=-\sum h_{i j} \theta_{j}+x_{i} w_{n+1}
$$

onde $x_{i}=\left\langle\nabla_{N} N, e_{i}\right\rangle$. Portanto, derivando a 1-forma $w_{n+1}$, obtemos

$$
d w_{n+1 i}=-\sum\left\{d h_{i j} \wedge w_{j}+h_{i j} d w_{j}\right\}+d x_{i} \wedge w_{n+1}+x_{i} d w_{n+1}
$$

Por outro lado, usando as equações de estrutura, obtemos

$$
d w_{n+1 i}=\sum w_{n+1 C} \wedge w_{C i}+\Omega_{n+1 i}
$$

Igualando as duas expressões acima e somando as expressões resultantes deduzimos que

$$
\operatorname{div}\left(\nabla_{N} N\right)=-n d H(N)+\|B\|^{2}+n \operatorname{Ric}(N)
$$


1.2.3 Proposição. Seja $F$ uma folheação de classe $C^{\infty}$, de codimensão um e transversalmente orientável definida sobre a variedade riemanniana completa $M^{n+1}$ e seja $N$ um campo de vetores unitário ortogonal às folhas de $F$ definido em toda a variedade $M$. Então, temos a seguinte desigualdade

$$
d H(N) \geq \operatorname{Ric}(N)-\frac{1}{n} \operatorname{div}\left(\nabla_{N} N\right)+H^{2}
$$

Demonstração. Aplicamos a desigualdade de Cauchy-Schwarz aos seguintes vetores do $\mathbb{R}^{n}$

$$
(1,1, \ldots, 1) \text { e }\left(h_{11}, h_{22}, \ldots, h_{n n}\right)
$$

Assim, obtemos

$(n H)^{2}=\left(h_{11}+h_{22}+\ldots+h_{n n}\right)^{2} \leq n\left(h_{11}^{2}+h_{22}^{2}+\ldots+h_{n n}^{2}\right) \leq n\|B\|^{2}$

Portanto, $n H^{2} \leq\|B\|^{2}$. Aplicando esta última desigualdade na expressão da Proposição 1.2.2, chegamos ao resultado

\subsection{Resultados Principais.}

O nosso objetivo é demonstrar o seguinte teorema que estabelece um limite superior para a função curvatura média de uma Folheação Transversalmente Orientável $F$. 
1.3.1 Teorema. Seja F uma folheação de classe $C^{\infty}$, codimensão um e transversalmente orientável, definida sobre uma variedade riemanniana completa $M$. Então, se $N$ denota o campo unitário de $M$ ortogonal às folhas de $F$, temos

$$
\begin{aligned}
& \text { (i) } \inf _{M}\left\{\operatorname{Ric}(N)-\frac{1}{n} \operatorname{div}\left(\nabla_{N} N\right)\right\} \leq 0 \\
& \text { (ii) } H^{2} \leq-\inf _{M}\left\{\operatorname{Ric}(N)-\frac{1}{n} \operatorname{div}\left(\nabla_{N} N\right)\right\}
\end{aligned}
$$

Demonstração. Suponha, por absurdo, que

$$
\operatorname{Ric}(N)-\frac{1}{n} \operatorname{div}\left(\nabla_{N} N\right)>0
$$

em todo o ponto de $M$. Pela Proposição 1.2.3, podemos dizer que vale a seguinte desigualdade em $M$

$$
d H(N)>H^{2}
$$

Seja agora $\gamma(s)$ uma curva integral do campo unitário $N$. Como a variedade $M$ é completa, o parâmetro $s$ varia em todo o $\mathbb{R}$ e portanto, sobre esta curva, a desigualdade acima pode ser escrita na forma

$$
\left(H_{\gamma(s)}\right)^{\prime}>H_{\gamma(s)}^{2} \quad \forall s \in \mathbb{R}
$$


Podemos escolher o campo $N$ de tal forma que $H_{\gamma(0)} \geq 0$ mudando, caso necessário, $N$ por $-N$. Esta mudança não altera a expressão

$$
\operatorname{Ric}(N)-\frac{1}{n} \operatorname{div}\left(\nabla_{N} N\right)
$$

e nem a desigualdade da Proposição 1.2.3. Desta maneira, temos que as desigualdades

$$
\left(H_{\gamma(s)}\right)^{\prime}>0, \quad H_{\gamma(s)}>0 \quad e \quad \frac{\left(H_{\gamma(s)}\right)^{\prime}}{H_{\gamma(s)}^{2}}>1
$$

são válidas para todo $s>0$. Considere agora a função real $G$ dada por

$$
G(s)=-\frac{1}{H_{\gamma(s)}}, \quad s>0
$$

Fixando um número estritamente positivo $b$ e aplicando o Teorema do Valor Médio para a função $G$ no intervalo $[b, s]$, obtemos

$$
-\frac{1}{H_{\gamma(s)}}+\frac{1}{H_{\gamma(b)}}=\frac{\left(H_{\gamma(c)}\right)^{\prime}}{H_{\gamma(c)}^{2}}(s-b)
$$

onde $c \in(b, s)$. Assim, a seguinte desigualdade é verdadeira para todo $s>b$

$$
-\frac{1}{H_{\gamma(s)}}+\frac{1}{H_{\gamma(b)}}>s-b
$$

Mas isto nos leva a uma contradição pois, fazendo $s \longrightarrow+\infty$, o lado direito desta desigualdade é ilimitado enquanto que o lado esquerdo é 
limitado. Portanto, podemos concluir que existe um ponto em $M$ no qual a função $\operatorname{Ric}(N)-\frac{1}{n} \operatorname{div}\left(\nabla_{N} N\right)$ é negativa e assim demonstramos que vale a afirmação do item $(i)$.

Para demonstrar o item (ii), supomos por absurdo que

$$
H_{p}^{2}>-\inf _{M}\left\{\operatorname{Ric}(N)-\frac{1}{n} \operatorname{div}\left(\nabla_{N} N\right)\right\}
$$

em algum ponto $p \in M$. Pelo item $(i)$, podemos afirmar que, para algum $a \geq 0$

$$
\inf _{M}\left\{\operatorname{Ric}(N)-\frac{1}{n} \operatorname{div}\left(\nabla_{N} N\right)\right\}=-a^{2}
$$

Vamos considerar o caso $a>0$. Da nossa hipótese concluímos que $H_{p}^{2}-a^{2}>0$. Considere agora, $\gamma(s)$ a curva integral de $N$ tal que $\gamma(0)=p$. Como foi feito no item $(i)$, podemos escolher uma direção $N$ tal que $H_{p}=H_{\gamma(0)} \geq 0$ e portanto, $H_{\gamma(0)}>a$. Por continuidade, existe um intervalo maximal $[0, b)$ onde

$$
H_{\gamma(s)}^{2}-a^{2}>0 \quad \forall s \in[0, b)
$$

Afirmação: $\quad b=+\infty$

De fato, se $b$ fosse finito, deveríamos ter $H_{\gamma(b)}^{2}=a^{2}$. Mas, pela Proposição 1.2.3

$$
\left(H_{\gamma(s)}\right)^{\prime} \geq H_{\gamma(s)}^{2}-a^{2}>0 \quad \forall s \in[0, b)
$$


concluindo que $H_{\gamma(s)}$ é uma função estritamente crescente em $[0, b]$, $H_{\gamma(0)}>a>0$ e $H_{\gamma(b)}=a$ o que é uma contradição.

Deste modo, podemos afirmar que para todo $s>0$ são válidas as seguintes desigualdades

$$
H_{\gamma(s)}>a>0, \quad\left(H_{\gamma(s)}\right)^{\prime} \geq H_{\gamma(s)}^{2}-a^{2}>0 \quad \text { e } \frac{\left(H_{\gamma(s)}\right)^{\prime}}{H_{\gamma(s)}^{2}-a^{2}} \geq 1
$$

Considere agora a função

$$
G(s)=\frac{1}{2 a} \ln \left(\frac{H_{\gamma(s)}-a}{H_{\gamma(s)}+a}\right), \quad s>0
$$

Fixando um número estritamente positivo $b$ e aplicando o Teorema do Valor Médio para a função $G$ no intervalo $[b, s]$, obtemos um $c \in(b, s)$ tal que

$$
\frac{1}{2 a} \ln \left(\frac{H_{\gamma(s)}-a}{H_{\gamma(s)}+a}\right)-\frac{1}{2 a} \ln \left(\frac{H_{\gamma(b)}-a}{H_{\gamma(b)}+a}\right)=\frac{\left(H_{\gamma(c)}\right)^{\prime}}{H_{\gamma(c)}^{2}-a^{2}}(s-b)
$$

Podemos assim deduzir que a seguinte desigualdade é válida para todo $s>b$

$$
\frac{1}{2 a} \ln \left(\frac{H_{\gamma(s)}-a}{H_{\gamma(s)}+a}\right)-\frac{1}{2 a} \ln \left(\frac{H_{\gamma(b)}-a}{H_{\gamma(b)}+a}\right) \geq s-b
$$

Obtemos uma contradição fazendo $s \longrightarrow+\infty$ : o lado esquerdo é limitado enquanto que o lado direito é ilimitado. Assim, devemos concluir que não existe um ponto $p \in M$ tal que $H_{p}^{2}>a^{2}$ demonstrando o item 
(ii) para o caso $a>0$. O caso $a=0$ nos leva a um argumento análogo ao do item $(i)$

1.3.2 Corolário. Seja $F$ uma folheação de classe $C^{\infty}$, codimensão um e transversalmente orientável definida sobre uma variedade riemanniana completa $M$ de curvatura seccional constante $\kappa$. Então, se o campo unitário $N$ ortogonal às folhas de $F$ é tal que $\operatorname{div}\left(\nabla_{N} N\right)=0$, temos que

$$
\begin{aligned}
& \text { (i) } \kappa \leq 0 \\
& \text { (ii) } H^{2} \leq-\kappa
\end{aligned}
$$

1.3.3 Corolário. Não existe uma folheação $F$ de classe $C^{\infty}$, codimensão um e transversalmente orientável definida sobre a variedade riemanniana completa $M$ de curvatura seccional constante $\kappa>0$ e tal que o campo unitário $N$, ortogonal às folhas de $F$, seja geodésico.

1.3.4 Definição. Uma Folheação $F$ definida em uma variedade riemanniana $M$ é dita Totalmente Geodésica se cada folha de $F$ é uma subvariedade totalmente geodésica de $M$. 
1.3.5 Teorema. Seja F uma folheação de classe $C^{\infty}$, codimensão um e transversalmente orientável definida sobre uma variedade riemanniana completa $M$. Então, se $N$ é o campo unitário de $M$ ortogonal às folhas de $F$, temos que

$$
F \text { é Totalmente Geodésica } \Longleftrightarrow \inf _{M}\left\{\operatorname{Ric}(N)-\frac{1}{n} \operatorname{div}\left(\nabla_{N} N\right)\right\}=0
$$

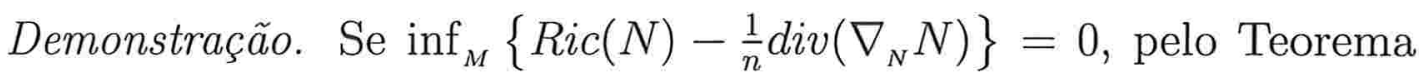
1.3.1 temos que $H=0$. Mas pela Proposição 1.2.3, $0 \geq \operatorname{Ric}(N)-$ $\frac{1}{n} \operatorname{div}\left(\nabla_{N} N\right)$ e assim conclui-se que $\operatorname{Ric}(N)-\frac{1}{n} \operatorname{div}\left(\nabla_{N} N\right)=0$ em $M$. Pela Proposição 1.2.2, temos $\|B\|^{2}=0$. A recíproca segue imediatamente da Proposição 1.2.2

1.3.6 Corolário. Seja $F$ uma folheação de classe $C^{\infty}$, codimensão um e transversalmente orientável definida sobre uma variedade riemanniana completa $M$. Se F possui uma folha compacta $L$ tal que, sobre esta folha, $\operatorname{Ric}(N) \geq 0$ e a curvatura média é constante e igual a $H_{L}=\left(-\inf _{M}\left\{\operatorname{Ric}(N)-\frac{1}{n} \operatorname{div}\left(\nabla_{N} N\right)\right\}\right)^{\frac{1}{2}}$. Então, F é totalmente geodésica. 
Demonstração. Pelo Teorema 1.3.1, a curvatura média da folha $L$ é a maior possível e então $d H(N)=0$ sobre a folha $L$. Portanto, pela Proposição 1.2.2, temos que a equação $\operatorname{div}\left(\nabla_{N} N\right)=\|B\|^{2}+$ $n \operatorname{Ric}(N)$ é válida sobre toda a folha $L$. Integrando esta equação sobre $L$, usando a hipótese $\operatorname{Ric}(N) \geq 0$ e o Teorema da Divergência concluímos que $\|B\|=0$ e $\operatorname{assim} L$ é totalmente geodésica. Logo, $\inf _{M}\left\{\operatorname{Ric}(N)-\frac{1}{n} \operatorname{div}\left(\nabla_{N} N\right)\right\}=0$ e pelo Teorema 1.3.5, a folheação $F$ é Totalmente Geodésica

\subsection{Exemplos no Espaço Hiperbólico.}

A seguir, vamos exibir alguns exemplos de folheações transversalmente orientáveis no Espaço Hiperbólico. Pelo Corolário 1.3.2, se o campo $N$ for geodésico, devemos ter $|H| \leq 1$. Vamos utilizar o modelo de espaço dado por

$$
\mathbb{H}^{3}=\left\{(x, y, z) \in \mathbb{R}^{3} ; z>0\right\} \quad d s^{2}=\frac{d x^{2}+d y^{2}+d z^{2}}{z^{2}}
$$




\subsubsection{Exemplo. A Folheação Trivial}

Considere a Folheação $F$ do espaço hiperbólico dada pela seguinte família de planos

$$
z=c, \quad c>0
$$

Cada folha desta folheação tem curvatura média constante $H=1$. O campo $N$ é geodésico e as suas curvas integrais são dadas por $\gamma(s)=\left(a, b, e^{s}\right)$ com $s \in \mathbb{R}$ e $(a, b) \in \mathbb{R}^{2}$.

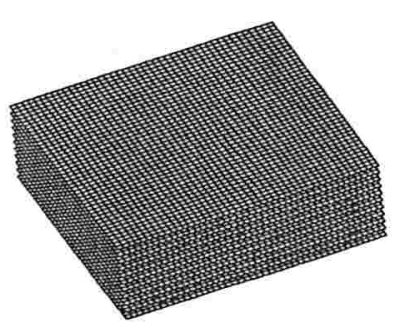

1.4.2 Exemplo. A Folheação por Horoesferas

Esta folheação é dada pela seguinte família de esferas

$$
x^{2}+y^{2}+(z-a)^{2}=a^{2}, \quad a>0
$$

Neste caso, o campo normal $N$ também é geodésico e as suas curvas integrais são as geodésicas do $\mathbb{H}^{3}$ cujo $\omega$-limite é a origem 


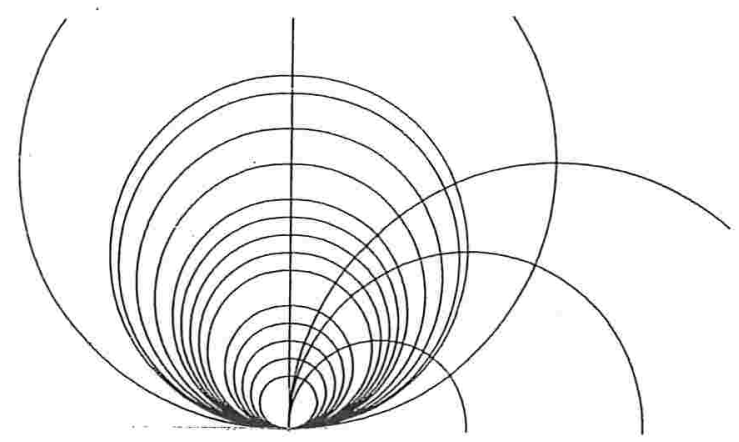

1.4.3 Exemplo. O Feixe de Planos

É a Folheação do $\mathbb{H}^{3}$ pela seguinte família de planos

$$
z-c y=0, \quad c \neq 0
$$

O campo normal $N$ é geodésico e as respectivas curvas integrais são dadas pelas geodésicas do $\mathbb{H}^{3}$ que são paralelas ao plano $z y$.

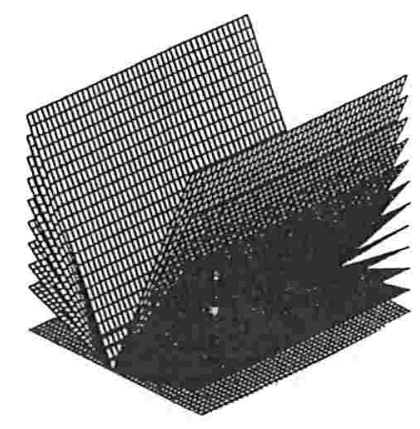


1.4.4 Exemplo. Folheação totalmente geodésica

Considere a folheação do $\mathbb{H}^{3}$ dada pela seguinte família de esferas

$$
x^{2}+y^{2}+z^{2}=c^{2}, \quad c>0
$$

Esta folheação é totalmente geodésica, o campo $N$ não é geodésico e tem curvas integrais que são retas que passam pela origem

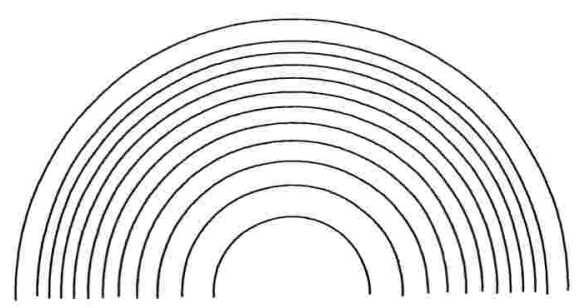

\subsection{Folheações Totalmente Umbílicas.}

Observando os exemplos obtidos anteriormente, notamos que as folhas das folheações exibidas eram subvariedades totalmente umbílicas do espaço hiperbólico. Obtemos a seguir uma condição suficiente sobre a folheação $F$ para que isto ocorra supondo a variedade ambiente $M$ com curvatura seccional constante. 
1.5.1 Teorema. Seja F uma folheação de classe $C^{\infty}$, de codimensão um, transversalmente orientável e definida sobre uma variedade riemanniana completa $M(\kappa)$ de curvatura seccional constante $\kappa \leq 0$. Se o campo $N$ ortogonal às folhas de $F$ for geodésico e se uma das folhas de F for totalmente umbílica então todas as folhas de F serão totalmente umbilicas.

Demonstração. Da demonstração da Proposição 1.2.2, obtemos a seguinte equação

$$
\begin{array}{r}
\left(d x_{i}+\sum x_{j} w_{j i}\right)\left(e_{k}\right)=x_{i} x_{k}+\sum h_{i j} h_{j k}+R_{n+1 i n+1 k}- \\
-\left(d h_{i k}+\sum h_{j k} w_{j i}+\sum h_{i j} w_{j k}\right)\left(e_{n+1}\right)
\end{array}
$$

onde $x_{i}=\left\langle\nabla_{N} N, e_{i}\right\rangle$. Supondo a segunda forma fundamental $\|B\|$ diagonalizada e usando as hipóteses de que $N$ é geodésico e de que $M$ tem curvatura seccional constante $\kappa$, temos que as curvaturas principais $h_{i i}$ satisfazem a seguinte equação diferencial

$$
h_{i i}^{2}+\kappa-d h_{i i}(N)=0
$$

Assim, se uma folha da folheação $F$ for totalmente umbílica, teremos uma condição inicial comum para a equação diferencial acima e portanto, pela unicidade de soluções, segue que $h_{11}=h_{22}=\ldots=h_{n n}$ 


\section{Capítulo 2}

\section{Folheações Rotacionais}

\subsection{Introdução.}

O nosso objetivo neste capítulo é determinar as Folheações Rotacionais do espaço hiperbólico $\mathbb{H}^{3}$ que possuem curvatura média constante $H$ tal que $0 \leq H \leq 1$. Por Folheação Rotacional entendemos folheações que são invariantes pela ação do grupo $O(2)$. Mostraremos ainda que não existem tais folheações na esfera euclidiana $\mathbb{S}^{3}$. Em todo este capítulo, usaremos o mesmo modelo de espaço hiperbólico que foi utilizado na seção 1.4. 


\subsection{Preliminares.}

Seja $G$ o grupo das isometrias do $\mathbb{H}^{3}$ que deixam a geodésica $x=y=0$ fixada pontualmente. O grupo $G$ age sobre $\mathbb{H}^{3}$ de forma natural por meio da aplicação

$$
(g, p) \in G \times \mathbb{H}^{3} \longmapsto g(p) \in \mathbb{H}^{3}
$$

As órbitas desta ação são de dois tipos:

(i) Pontos fixos (órbitas de pontos da geodésica fixada)

(ii) Órbitas homeomorfas ao $\mathbb{S}^{1}$

2.2.1 Definição. O Espaço de Órbitas é o conjunto quociente do $\mathbb{H}^{3}$ pela relação de equivalência $\equiv$ definida por

$$
p \equiv q \Longleftrightarrow \exists g \in G / g(p)=q
$$

Neste caso, podemos identificar o espaço de órbitas com o seguinte subconjunto do plano euclideano: $\mathbb{H}^{3} / G=\left\{(y, z) \in \mathbb{R}^{2} ; y \geq 0, z>0\right\}$ 
Podemos transformar este subconjunto em uma variedade riemanniana bastando para isto introduzir a Métrica da Distância Orbital, isto é, a distância entre dois pontos no espaço de órbitas $\mathbb{H}^{3} / G$ é, por definição, a distância entre suas respectivas órbitas no $\mathbb{H}^{3}$. Assim, a métrica no $\mathbb{H}^{3} / G$ é dada por $d^{2}=\frac{d y^{2}+d z^{2}}{z^{2}}$.

2.2.2 Definição. Uma hipersuperfície $M$ do $\mathbb{H}^{3}$ é dita Rotacional se vale o seguinte

$$
p \in M \Longrightarrow g(p) \in M, \quad \forall g \in G
$$

2.2.3 Definição. Se $\pi: \mathbb{H}^{3} \longrightarrow \mathbb{H}^{3} / G$ é a aplicação quociente e $M$ é uma hipersuperfície rotacional do $\mathbb{H}^{3}$ então $\pi(M)$ é chamada de Curva Geradora de M.

O nosso objetivo é exibir folheações do $\mathbb{H}^{3}$ por hipersuperfícies rotacionais de curvatura média constante. Isto será feito mediante uma análise das curvas geradoras do espaço de órbitas. O primeiro passo será definir um novo sistema de coordenadas para $\mathbb{H}^{3} / G$. 


\subsection{Um sistema de coordenadas para $\mathbb{H}^{3} / G$.}

Considere um ponto $(y, z) \in \mathbb{H}^{3} / G$. Existe uma única geodésica parametrizada pelo comprimento de arco, digamos $\eta$, que passa por este ponto e é ortogonal ao eixo $z$. Esta geodésica determina um par de valores $(r, s)$ definidos por

$$
\begin{aligned}
\left(0, e^{r}\right) & =\text { interseç̧ão da geodésica } \eta \text { com o eixo } z \\
s & =\text { distância do ponto }(y, z) \text { ao eixo } z
\end{aligned}
$$

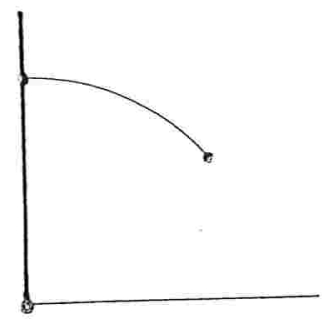

É fácil de ver que $(r, s)$ determina um sistema de coordenadas em $\mathbb{H}^{3} / G$ e a transformação de mudança de coordenadas é dada por

$$
\left\{\begin{array}{l}
y=e^{r} \tanh (s) \\
z=e^{r} \operatorname{sech}(s)
\end{array}\right.
$$

Assim, podemos determinar as expressões para $d y$ e $d z$

$$
\left\{\begin{array}{l}
d y=e^{r} \tanh (s) d r+e^{r} \operatorname{sech}^{2}(s) d s \\
d z=e^{r} \operatorname{sech}(s) d r-e^{r} \tanh (s) \operatorname{sech}(s) d s
\end{array}\right.
$$


Desta forma, podemos expressar a métrica em $\mathbb{H}^{3} / G$ no sistema de coordenadas $(r, s)$

$$
d^{2}=\cosh ^{2}(s) d r^{2}+d s^{2}
$$

De posse deste novo sistema de coordenadas, vamos estabelecer uma relação que caracteriza as curvas geradoras das superfícies de rotação de curvatura média constante. Seja então $\gamma=(r(t), s(t))$ uma curva parametrizada pelo comprimento de arco em $\mathbb{H}^{3} / G$ e que é uma curva geradora de uma superfície de rotação do $\mathbb{H}^{3}$, digamos $S$, que possui curvatura média constante. A seguinte fórmula é obtida em [2]

$$
2 H=\kappa_{\gamma}-\frac{d}{d n} \ln (v(r, s))
$$

onde:

$\mathrm{v}(\mathrm{r}, \mathrm{s})$ é o comprimento $\left(e m \mathbb{H}^{3}\right)$ da órbita $\pi^{-1}(r, s)$

$\kappa_{\gamma}$ é a curvatura geodésica de $\gamma$ e

$n$ é a normal unitária de $\gamma$.

Observe que $v(r, s)=\int_{0}^{2 \pi}\left\|\beta^{\prime}(t)\right\| d t$, onde $\beta(t)=(y \cos (t), y \operatorname{sen}(t), z)$. Obtemos então que $v(r, s)=2 \pi \operatorname{senh}(s)$. Por outro lado, denotando os vetores tangentes às curvas coordenadas $r=$ cte. e $s=c t e$. por $\frac{\partial}{\partial s}$ e por $\frac{\partial}{\partial r}$ respectivamente, temos

$$
\gamma^{\prime}=\operatorname{sen}(\alpha) e_{r}+\cos (\alpha) e_{s}
$$


onde:

$\alpha=$ ângulo entre $\gamma^{\prime}$ e $\frac{\partial}{\partial s}$

$e_{r}=\frac{1}{\cosh (s)} \frac{\partial}{\partial r}$ e $e_{s}=\frac{\partial}{\partial s}$

e podemos escrever então $n=(\cos (\alpha)) e_{r}-(\operatorname{sen}(\alpha)) e_{s}$.

Calculando a curvatura geodésica de $\gamma$ obtemos $\kappa_{\gamma}=\alpha^{\prime}+r^{\prime} \operatorname{senh}(s)$ e assim, a fórmula $(*)$ pode ser escrita como

$$
2 H=\alpha^{\prime}+r^{\prime} \operatorname{senh}(s)+\frac{\operatorname{sen}(\alpha) \cosh (s)}{\operatorname{senh}(s)}
$$

Observando ainda que podemos escrever $\frac{d r}{d t}=\frac{\operatorname{sen}(\alpha)}{\cosh (\alpha)}$ e $\frac{d s}{d t}=\cos (\alpha)$, a fórmula $(*)$ resultará em

$$
\operatorname{senh}(s) \cosh (s) \operatorname{sen}(\alpha)-H \operatorname{senh}^{2}(s)=c t e .
$$

Logo, a equação acima caracteriza as curvas geradoras das superfícies de rotação com curvatura média constante.

\subsection{Folheações do espaço hiperbólico $\mathbb{H}^{3}$.}

Vamos encontrar todas as folheações rotacionais com curvatura média constante do $\mathbb{H}^{3}$ através da análise das curvas geradoras no espaço de órbitas. Neste sentido, a seguinte proposição é natural 
2.4.1 Proposição. Seja $F^{\prime}=\left\{\gamma_{\lambda}\right\}_{\lambda \in \Lambda}$ uma folheação de codimensão um do espaço de órbitas $\mathbb{H}^{3} / G$ tal que: (1) Cada folha que intercepta o eixo $r$ o faz ortogonalmente (2) Cada folha $\gamma_{\lambda}=(r, s)$ satisfaz a equação $(* *)$ para um certo $H_{\lambda}$ constante. Então a folheação $F^{\prime}$ induz uma folheação $F=\left\{M_{\lambda}\right\}_{\lambda \in \Lambda}$ do $\mathbb{H}^{3}$ por superfícies de rotação que possuem curvatura média constante e cada $M_{\lambda}$ tem $\gamma_{\lambda}$ como curva geradora.

Deste modo, ao analisar folheações do $\mathbb{H}^{3} / G$ que satisfazem a proposição anterior, teremos obtido as folheações rotacionais de curvatura média constante do $\mathbb{H}^{3}$. Vejamos alguns exemplos:

\subsubsection{Exemplo: Caso $H=0$ e $c t e=0$ na equação (**).}

Neste caso devemos ter $\gamma^{\prime}=(\operatorname{sen}(\alpha)) e_{r}+(\cos (\alpha)) e_{s}=(0,1)$ e então $\gamma(t)=\left(t_{0}, t\right)$
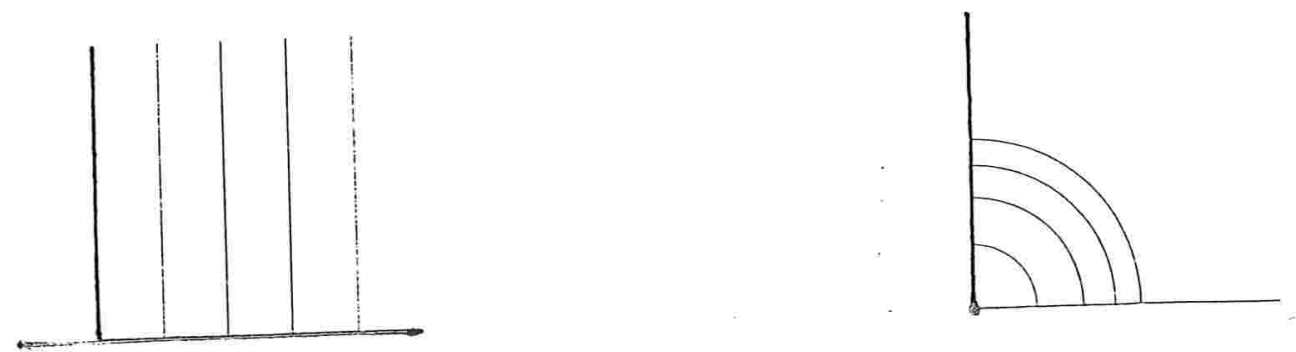
Portanto obtemos uma folheação do $\mathbb{H}^{3}$ por superfícies de rotação com $H=0$.

2.4.3 Exemplo: Caso $H=1$ e cte $=0$ na equação (**).

Neste caso a equação $(* *)$ fica com a forma $\operatorname{sen}(\alpha)=\tanh (s)$. Mas temos ainda que

$$
\frac{d s}{d r}=\frac{\cosh (s) \cos (\alpha)}{\operatorname{sen}(\alpha)}=\frac{\cosh (s)}{\tanh (s)}\left( \pm \sqrt{1-\tanh ^{2}(s)}\right)= \pm \frac{\cosh (s)}{\operatorname{senh}(s)}
$$

e então $r= \pm \ln (\cosh (s))+$ cte. Passando ao sistema de coordenadas $(y, z)$, estas folheações correspondem à folheação trivial e à folheação por horoesferas
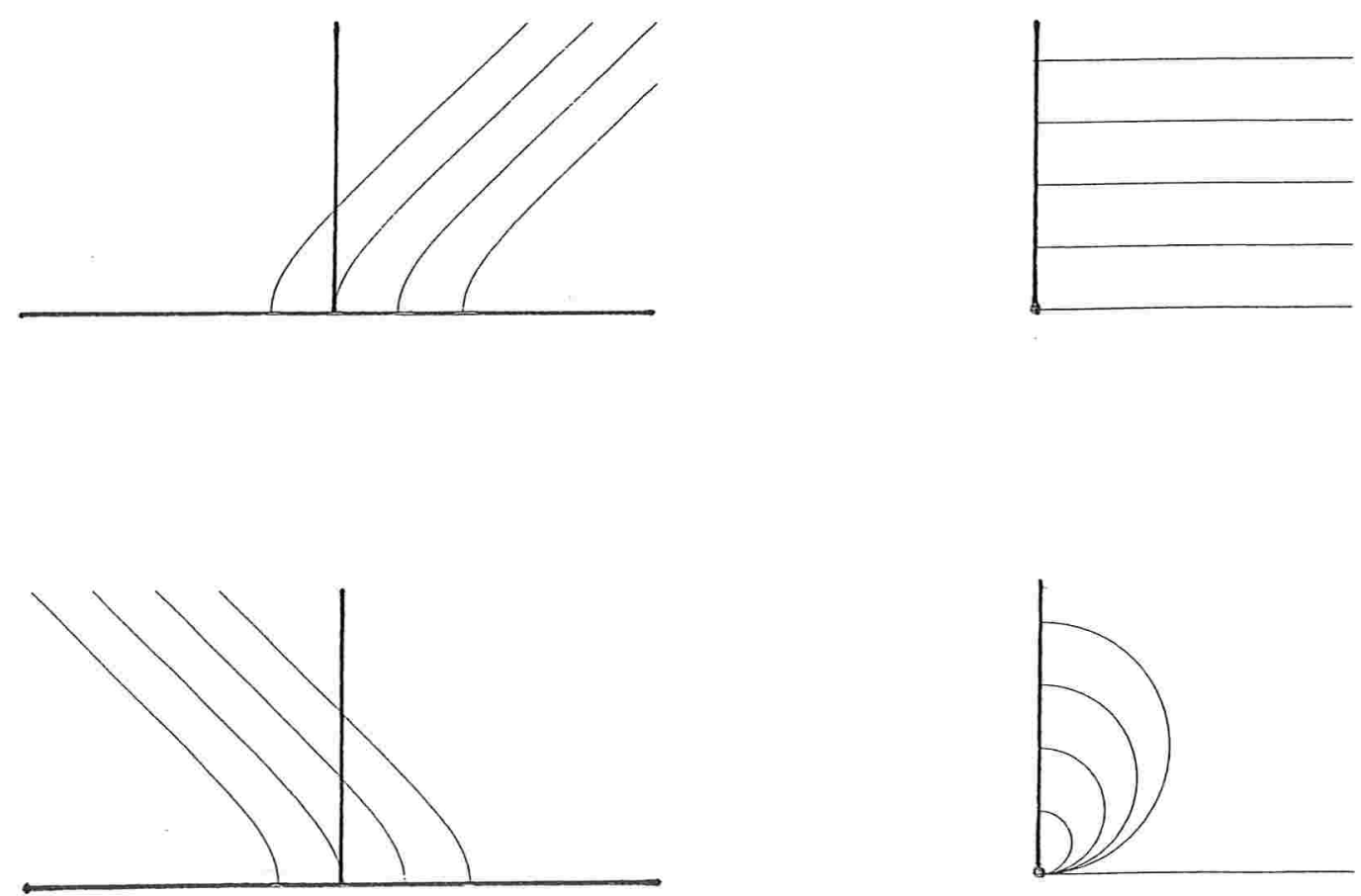
2.4.4 Exemplo: Caso $0<H<1$ na equação $(* *)$ e cte $=0$.

Neste caso a equação $(* *)$ fica na forma

$$
\operatorname{senh}(s) \cosh (s) \operatorname{sen}(\alpha)-H \operatorname{senh}^{2}(s)=0
$$

fazendo $\operatorname{senh}(s) \neq 0$ obtemos

$$
\operatorname{sen}(\alpha)=H \tanh (s)
$$

e lembrando que

$$
\frac{d s}{d r}=\frac{\cosh (s) \cos (\alpha)}{\operatorname{sen}(\alpha)}
$$

deduzimos que

$$
d r=\frac{ \pm H \operatorname{senh}(s)}{\cosh ^{2}(s) \sqrt{1-H \tanh ^{2}(s)}} d s
$$

e integrando

$$
r= \pm \ln \left(\frac{1}{\cosh (s)}+\sqrt{\frac{1}{\cosh ^{2}(s)}+\frac{1-H^{2}}{H^{2}}}\right)+c t e .
$$

Passando para o sistema de coordenadas $(y, z)$, vemos que estas folheações são obtidas girando ao redor do eixo $z$, a seguinte família de circunferências

$$
y^{2}+(z-\sqrt{a})^{2}=\left(\frac{\sqrt{a}}{H}\right)^{2}
$$

onde $H \in(0,1)$ e $a>0$. Temos então duas posibilidades de folheação: $H$ fixo e $a>0$ variável ou então $H$ variável e $a>0$ fixo. 
Observamos ainda que a constante na equação $(* *)$ deve ser sempre nula a fim de que possamos obter folheações sem singularidades.
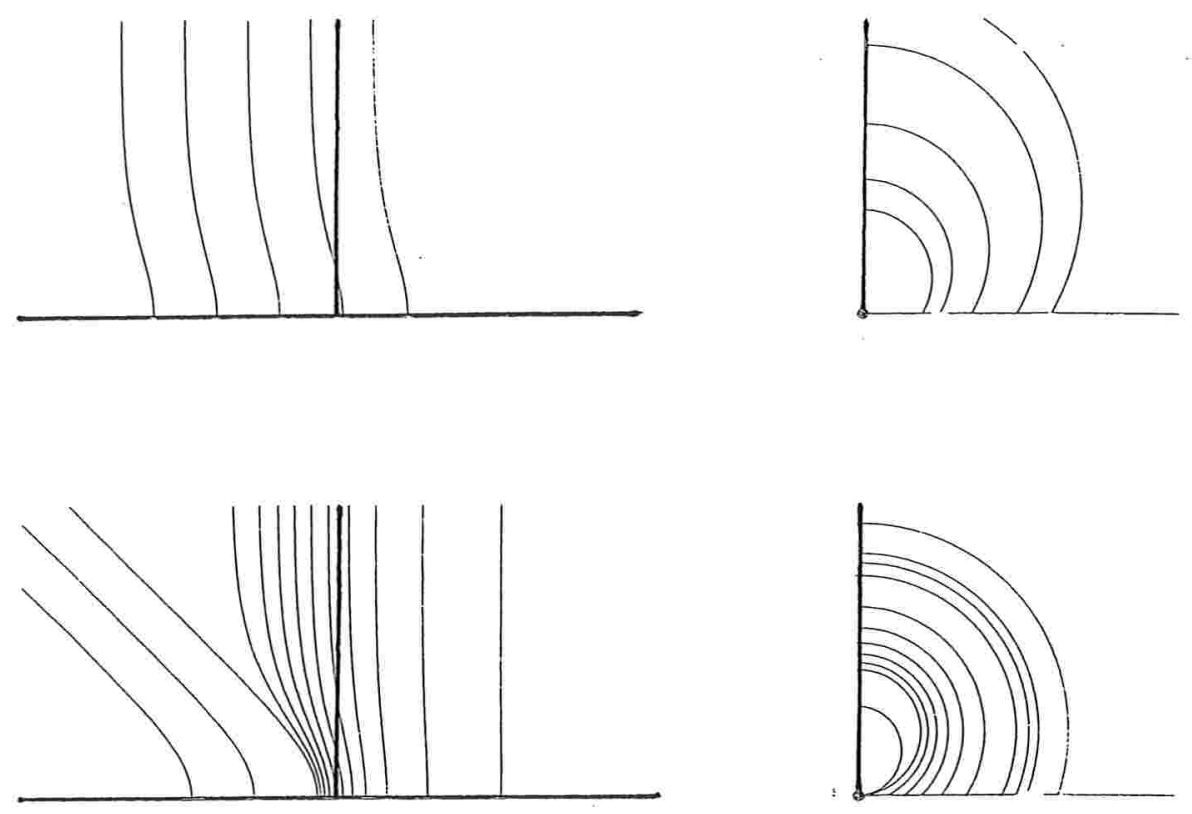

\subsection{A não-existência de uma folheação rotacional na esfera euclidiana $\mathbb{S}^{3}$.}

Seja $\mathbb{S}^{3}=\left\{\left(x_{1}, x_{2}, x_{3}, x_{4}\right) \in \mathbb{R}^{4} ; \sum x_{i}^{2}=1\right\}$ a esfera euclideana e seja $G$ o grupo de isometrias do $\mathbb{S}^{3}$ que deixa a geodésica $x_{1}^{2}+x_{2}^{2}=1$ fixada pontualmente. Um elemento $g \in G$ pode ser representado por uma matriz da forma 


$$
\left(\begin{array}{ll}
I & 0 \\
0 & \sigma
\end{array}\right)
$$

onde $I$ é a matriz identidade $2 \times 2$ e $\sigma \in O(2)$. Este grupo age sobre $\mathbb{S}^{3}$ através da aplicação

$$
(g, p) \in G \times \mathbb{S}^{3} \longmapsto g(p) \in \mathbb{S}^{3}
$$

As órbitas desta ação são de dois tipos: pontos ou órbitas homeomorfas ao $\mathbb{S}^{1}$

O espaço de órbitas, denotado por $\mathbb{S}^{3} / G$, é o espaço quociente pela relação de equivalência $\equiv$ sobre $\mathbb{S}^{3}$ definida por

$$
p \equiv q \Longleftrightarrow \exists g \in G / g(p)=q
$$

Este espaço de órbitas pode ser identificado com o hemisfério fechado 2-dimensional

$$
S_{+}^{2}=\left\{\left(x_{1}, x_{2}, x_{3}, 0\right) \in \mathbb{S}^{3} ; x_{3} \geq 0\right\}
$$

e a aplicação natural $\pi: \mathbb{S}^{3} \longrightarrow S_{+}^{2}$ é dada por

$$
\pi\left(x_{1}, x_{2}, x_{3}, x_{4}\right)=\left(x_{1}, x_{2}, \sqrt{x_{3}^{2}+x_{4}^{2}}, 0\right)
$$

(veja, por exemplo, a referência [2]). 
Assim como foi feito na seção 2.3, uma hipersuperfície rotacional $M$ satisfaz $p \in M \Longrightarrow g(p) \in M$ e as curvas geradoras são dadas por $\gamma=\pi(M)$.

2.5.1 Teorema. Não existe uma folheação de codimensão um da esfera euclideana $\mathbb{S}^{3}$ tal que todas as suas folhas sejam hipersuperfícies de rotação.

Demonstração. Seja $F=\left\{M_{\alpha}\right\}_{\alpha \in \Lambda}$ uma folheação de codimensão um do $\mathbb{S}^{3}$ tal que cada folha $M_{\alpha}$ é uma hipersuperfície de rotação. A família $\left\{\pi\left(M_{\alpha}\right)\right\}_{\alpha \in \Lambda}$ é uma partição do hemisfério $S_{+}^{2}$. De fato, se $p \in S_{+}^{2}$ então existe $\alpha \in \Lambda$ tal que $p \in \pi\left(M_{\alpha}\right)$ pois $F$ é uma folheação do $\mathbb{S}^{3}$ e $\pi$ é uma função sobrejetora. Por outro lado, se $p=\pi\left(m_{\alpha}\right)=\pi\left(m_{\beta}\right)$, onde $m_{\alpha} \in M_{\alpha}$ e $m_{\beta} \in M_{\beta}$, então pela definição da relação de equivalência $\equiv$, existe $g \in G$ tal que $g\left(m_{\alpha}\right)=m_{\beta}$. Mas $M_{\alpha}$ é uma hipersuperfície de rotação para todo $\alpha \in \Lambda$. Portanto $g\left(m_{\alpha}\right)=m_{\beta} \in M_{\alpha}$ e então deduzimos que $\alpha=\beta$. O seguinte fato é agora evidente:

Seja $F$ uma folheação do $\mathbb{S}^{3}$ por hipersuperfícies de rotação. Então, $F$ induz uma folheação de classe $C^{\infty}$ e de codimensão um no interior do espaço de órbitas $S_{+}^{2}$, denotada por $F / \equiv$, cujas folhas são as curvas geradoras de cada folha da folheação $F$. 
Mas é bem conhecido que $F$ tem uma folha homeomorfa ao toro plano (veja [16]). Assim, a curva geradora desta folha é homeomorfa ao $\mathbb{S}^{1}$. Conclui-se então que a folheação $F / \equiv$ do interior do espaço de órbitas, tem uma folha compacta homeomorfa ao $\mathbb{S}^{1}$ contradizendo o Teorema de Poincaré-Bendixson do plano 


\section{Capítulo 3}

\section{Folheações Riemannianas}

\subsection{Introdução.}

Neste capítulo trataremos das folheações riemannianas e aplicaremos os resultados do primeiro capítulo ao estudo de tais folheações.

\subsection{Preliminares.}

Seja $M$ uma variedade Riemanniana completa de dimensão $n+1$ e $F$ uma folheação de codimensão um transversalmente orientável em $M$. Denotaremos por $T M$ o fibrado tangente de $M$ e por $L$ o fibrado tangente da folheação $F$. Temos então a seguinte decomposição 


$$
T M=L \oplus L^{\perp}
$$

onde $L^{\perp}$ é o fibrado normal de $F$ em TM, isto é, $L^{\perp}$ é o espaço gerado pelo campo $N$, ortogonal às folhas de $F$, no fibrado $T M$. Deste modo, podemos escrever a seguinte sequência exata

$$
0 \longrightarrow L \longrightarrow T M \stackrel{\pi}{\rightarrow} L^{\perp} \longrightarrow 0
$$

e portanto, para todo campo $Y$ definido em $p \in M$, temos decomposição única dada por

$$
Y_{p}=T_{p}+\lambda_{p} N_{p}
$$

onde $T \in L$.

Esta decomposição introduz naturalmente uma métrica em $L^{\perp}$

$$
g(\pi(Y), \pi(Z))=\langle Y, Z\rangle
$$

3.2.1 Definição. A Conexão de Bott em $L^{\perp}$ é a conexão dada por

$$
\stackrel{\circ}{\nabla}_{X} \pi(Y)=\pi([X, Y])
$$

onde $X \in L$ e $Y \in T M$. 
3.2.2 Definição. Uma folheação $F$ sobre $M$ é dita Riemanniana se $L^{\perp}$ está equipado com uma métrica $\bar{g}$ que possui a seguinte propriedade

$$
X \bar{g}(\pi(Y), \pi(Z))=\bar{g}\left(\stackrel{\circ}{\nabla}_{X} \pi(Y), \pi(Z)\right)+\bar{g}\left(\pi(Y), \stackrel{\circ}{\nabla}_{X} \pi(Z)\right)
$$

onde $X \in L$ e $Y, Z \in T M$.

3.2.3 Definição. A métrica Riemanniana $\langle$,$\rangle definida sobre M$ dita bundle like (ou $B L$ ) com respeito à folheação $F$ se a métrica $g$ induzida por $\langle$,$\rangle em L^{\perp}$, transforma a folheação $F$ em uma folheação Riemanniana, isto é,

$$
X g(\pi(Y), \pi(Z))=g\left(\stackrel{\circ}{\nabla}_{X} \pi(Y), \pi(Z)\right)+g(\pi(Y), \stackrel{\circ}{\nabla} \pi(Z))
$$

onde $X \in L$ e $Y, Z \in T M$.

O seguinte teorema é demonstrado em [17]

3.2.4 Teorema. Seja $(M,\langle\rangle,, \nabla)$ uma variedade riemanniana completa e F uma folheação de codimensão um transversalmente orientável de $M$ com normal unitária $N$. Então, $F$ é uma folheação riemanniana e a métrica $\langle$,$\rangle é B L$ com respeito à $F$ se, e só se,

$$
\left\langle\nabla_{Z} X, W\right\rangle+\left\langle\nabla_{W} X, Z\right\rangle=0
$$

onde $X \in L$ e $Z, W \in L^{\perp}$ 


\subsection{Resultados Principais}

3.3.1 Teorema. Seja F uma folheação riemanniana de codimensão um, transversalmente orientável de uma variedade riemanniana $(M,\langle\rangle$, com métrica BL com respeito à $F$. Então, se $N$ é o campo ortogonal à folheação $F$, temos que $N$ é um campo geodésico.

Demonstração. Basta mostrar que $\nabla_{N} N=0$. De fato, pelo torema 3.2.4, temos

$$
\left\langle\nabla_{N} X, N\right\rangle+\left\langle\nabla_{N} X, N\right\rangle=0
$$

e portanto $\left\langle\nabla_{N} X, N\right\rangle=0$ para todo $X \in L$. Por outro lado,

$$
\langle X, N\rangle=0 \Longrightarrow\left\langle\nabla_{N} X, N\right\rangle+\left\langle X, \nabla_{N} N\right\rangle=0
$$

assim, $\left\langle X, \nabla_{N} N\right\rangle=0$ para todo $X \in L$. Mas como $N$ é um campo unitário segue que $\left\langle\nabla_{N} N, N\right\rangle=0$. Logo, $\nabla_{N} N=0$

3.3.2 Corolário. Seja F uma folheação Riemanniana de codimensão um e transversalmente orientável, definida sobre uma variedade Riemanniana $(M,\langle\rangle$,$) tal que \langle$,$\rangle é B L$ com respeito à folheação $F$. Então,

F é totalmente geodésica $\Longleftrightarrow \inf _{M}\{\operatorname{Ric}(N)\}=0$ 
Demonstração. Segue do teorema anterior e do teorema 1.3.5

3.3.3 Corolário [19]. Seja $F$ uma folheação Riemanniana de codimensão um e transversalmente orientável, definida sobre a variedade riemanniana $(M,\langle\rangle$,$) tal que \langle$,$\rangle é B L$ com respeito à folheação $F$. Se $\operatorname{Ric}(N)<0$ em pelo menos um ponto de $M$, então $F$ não pode ser totalmente geodésica.

Demonstração. Segue imediatamente do corolário anterior

3.3.4 Corolário [19]. Seja F uma folheação Riemanniana de codimensão um e transversalmente orientável, definida sobre a variedade riemanniana $(M,\langle\rangle$,$) tal que \langle$,$\rangle é B L$ com respeito à folheação $F$. Se tivermos $\operatorname{Ric}(N) \geq\|B\|^{2}$, então $F$ é totalmente geodésica.

Demonstração. Pelo teorema 1.3.1 temos que $\inf _{M}\{\operatorname{Ric}(N)\} \leq 0$ e, pela hipótese, $\inf _{M}\{\operatorname{Ric}(N)\} \geq 0$. Portanto, $\inf _{M}\{\operatorname{Ric}(N)\}=0 \mathrm{e}$ assim, $F$ é totalmente geodésica pelo corolário 3.3.2 


\section{Bibliografia}

[1] Abe, K., Applications of a Riccati type differential equation to Riemannian manifolds with totally geodesic distributions, Tôhoku Math.J. 24, 425-440 (1973).

[2] Back, A., doCarmo, M.P., Hsiang, W.Y., On some Fundamental equations of Equivariant Riemannian geometry, Preprint, (1985).

[3] Barbosa, J.L.M., Kenmotsu, K., Oshikiri, G., Foliations by hipersurfaces with constant mean curvature, Math.Z. 207, 97-107 (1991).

[4] Brito, F.G.B., A remark on minimal foliations of codimension two, Tôhoku Math.J. 36, 341-350 (1984). 
[5] Brito, F.G.B., Walczak, P.G., Totally geodesic foliations with integrable normal bundle, Bol.Soc.Bras.Mat. 17, 41-46 (1986).

[6] Harvey, R., Lawson, H., Calibrated Foliations Amer.J.Math. 103, 411-435 (1981).

[7] Hsiang, W.Y., On generalization of theorems of A.P. Alexandrov and C. Delaunay on hypersurfaces of constant mean curvature, Duke Math.J. 49, 485-496 (1982).

[8] Meeks, W.H., The topology and geometry of embedded surfaces of constant mean curvature, J.Diff.Geom. 27, 539-552 (1988).

[9] Oshikiri, G., Some remarks on minimal foliations, Tôhoku Math.J. 39, 223-229 (1987).

[10] Oshikiri, G., A remark on minimal foliations, Tôhoku Math.J. 33, 133-137 (1981). 
[11] Oshikiri, G., On codimension-one foliations of constant mean curvature, Math.Z. 203, 105-113 (1990).

[12] Reinhart, B., Foliated manifolds with bundle-like metrics, Ann. of Math. 69, 119-132 (1959).

[13] Rovenskii, B.Yu., Totally geodesic foliations with nearly integrable orthogonal distribution, Sibirskii Matem. Zhurnal 32, 199-203 (1989).

[14] Rummler, H., Quelques notions simples en geométrie riemannienne et leurs applications aux feullietages compacts, Comment.Math.Helv. 54, 224-239 (1979).

[15] Sullivan, D., A homological characterization of foliations consisting of minimal surfaces, Comment.Math.Helv. 54, 218-223 (1979).

[16] Tamura, I., Topology of foliations: An introduction, Transl.of AMS, (1992). 
[17] Tondeur, P., Foliations on Riemannian Manifolds, Universitext, Springer-Verlag (1988).

[18] Tondeur, P., Kamber, F.W., Harmonic Foliations, Lecture Notes in Mathematics 949, 75-121 (1980).

[19] Tondeur, P., Vanhecke, L., Jacobi Fields, Riccati equation and Riemannian foliations, Illinois Math.J. 40, 211-225 (1996). 